\title{
Determination of Radium-226 in Rock Samples by Liquid Scintillation Counter
}

\author{
W. M. Abdellah \\ Radiation Protection Department, Nuclear and Radiological Regulatory Authority, Cairo, Egypt \\ Email:wmsra@yahoo.com
}

How to cite this paper: Abdellah, W.M. (2019) Determination of Radium-226 in Rock Samples by Liquid Scintillation Counter. Open Journal of Applied Sciences, 9, 270-284.

https://doi.org/10.4236/ojapps.2019.94023

Received: March 18, 2019

Accepted: April 23, 2019

Published: April 26, 2019

Copyright (c) 2019 by author(s) and Scientific Research Publishing Inc. This work is licensed under the Creative Commons Attribution International License (CC BY 4.0).

http://creativecommons.org/licenses/by/4.0/

(c) (i) Open Access

\begin{abstract}
The present work examines the validity of using barium as a carrier for radium-226 determination and optimizes the amount added during the radiochemical separation from rock samples. The activity concentration of Ra-226 is determined in rock samples by liquid scintillation spectrometer via its short-lived daughters after the radiochemical separation. The activity of Ra-226 was calculated based on the counts measured in a window set for Po-214 peak. The activity measured by Liquid Scintillation Counter, LSC using Pulse Shape Analysis, PSA. Ba-133 was used for chemical yield. The applied method was tested using a Certified Reference Material, CRM ore number-Dl-1a which shows a good agreement with the certified values. The applied method for measuring Ra-226 has been adapted to be used in rocks such as monazite. The average activity for Ra-226 in rock samples collected from the commercial grade Egyptian monazite was $13.85 \pm 2.05 \mathrm{~Bq} / \mathrm{g}$ with a detection limit $9.58 \mathrm{mBq} / \mathrm{g}$. The optimum conditions have been established during the present work such as precision, cost and time preparation which makes the method useful for radio chemists and the decision makers.
\end{abstract}

\section{Keywords}

Liquid Scintillation Counter, Spectrometry, Rock, Monazite, Radium-226

\section{Introduction}

Radium is an alkaline earth metal with the atomic number 88 , belonging to Group IIA in the Periodic table. It has more than 20 isotopes, all of them radioactive. Two radium isotopes, Ra-226 and Ra-228 are very significant from a radiological protection point of view due to their relatively long half-lives, presence in nature, and high dose conversion factors [1]. 
Radium has in the past and continues to be used in numerous applications including medical diagnosis and therapy, industrial applications such as selfluminous dials on clocks, instruments and is a waste product of uranium mining, and other natural resource exploitation processes Naturally Occurring Radioactive Material, NORM. Considering the radio-toxicity of different radionuclides, radium isotopes are important because they can be easily incorporated into bones due to having similar properties to other elements from Group II (i.e. calcium) and produce short living radionuclides of high specific activity [1]. The decay data for radium-226 isotopes are presented in Figure 1.

Uranium and Radium concentration in the groundwater strongly depends on the geological matrix and their concentrations water rock interaction mechanism. Generally, the determination of $\mathrm{Ra}$ and $\mathrm{U}$ isotopes are performed by $\alpha$-spectrometry, Liquid Scintillation Counter, LSC, and $\gamma$-spectrometry. Among them, $\alpha$-spectrometry is the most sensitive technique and allows simultaneous measurement of the radium and uranium isotopes [3] [4] [5]. However, chemical isolation of radium and uranium are not easy. Several studies have been carried out to study the radium concentrations in different matrix's [5].

The selection of the method for analysis of the environmental samples must be done in consideration the requirements for the user and requirements of legal acts regulating the content of radium isotopes. The most important task for every analyst is to choose the most appropriate and suitable analytical procedures for the analysis [6].

Many studies have been done on the detection of Ra-226 in water samples, which are geologically less challenging than other matrixes. The less LSC techniques has frequently been applied [7] [8] [9], and proven to be very successful in the accurate determination of low concentration of Ra-226. The Authors Saarinen \& Suksi [10] and Juutenen [11] have proved that the LSC technique can also provide useful results in the area under this investigation. In case of rock samples, radium needs to be chemically separated and purified from other contaminants isotopes using anion exchange chromatography. However, the resulting radium fraction usually far from pure, typically contain lead and barium sulphate, and small amounts from $\mathrm{Ti}, \mathrm{Nb}$, and $\mathrm{Zr}$. Additional purification steps

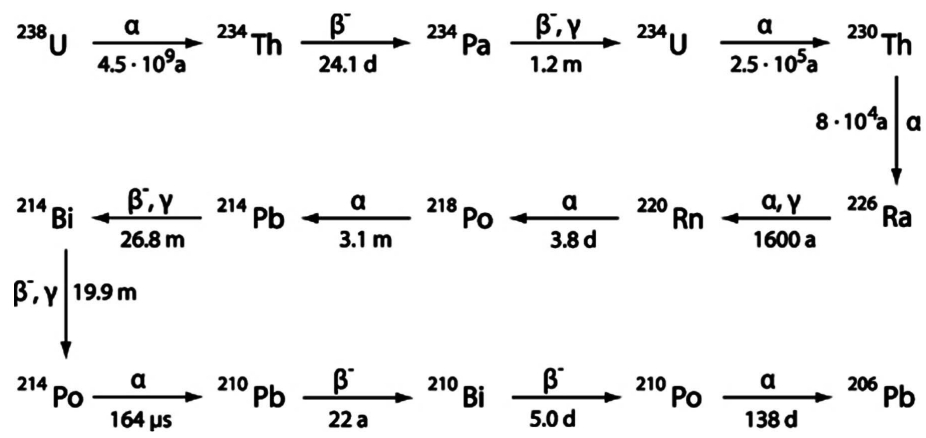

Figure 1. Simple decay scheme of U-238 series including only the major decay radionuclides [2]. 
are therefore necessary to obtain reliable results in the determination of Ra-226 activity as these interfering elements may because problems such as self-absorption and quenching [12].

The purpose of the present work is development of a method for the determination of Ra-226 from the rock samples using solid phase extraction technique. The measurement and applied techniques for sample source preparation optimized in order to achieve the lowest possible detection limits. The optimization of the applied method includes minimization of the background count rates as well as determination of the most favorable measurement conditions for liquid scintillation counter. Additionally, the obtained data will lead to a better understanding of the equilibrium in rock.

\section{Experimental}

\subsection{Apparatus and Chemicals}

Liquid scintillation counter, LSC from PerkinElmer 1220 Quantlus Ultra Low-Level and gamma ray spectrometer based on HPGe detector connected to a computerized multi-channel analyzer. All chemicals used were analytical, spectroscopic grade and used as received without any further purification and purchased from Sigma-Aldrich. Ba-133 tracer was prepared by diluting the stock solution till 1.66 $\mathrm{Bq} / \mathrm{ml}$. Ra-226 tracer a stock solution activity, $89 \pm 4 \mathrm{~Bq} / \mathrm{g}$ was diluted with 0.5 $\mathrm{M} \mathrm{HCl}$ to prepare the working solution $3.54 \mathrm{~Bq} / \mathrm{ml}$.

\subsection{Sample and Preparation}

Commercial grade monazite samples were supplied from the Egyptian Nuclear Materials Authority (ENMA). The mesh screen sizes of the samples under the present study were +50 to 100 mesh. The samples were decomposition using concentrated acids $4 \mathrm{ml} \mathrm{HNO}_{3}, 4 \mathrm{ml} \mathrm{HF}$ and $4 \mathrm{ml} \mathrm{HCl}$ to digest $50 \mathrm{mg}$ from the samples. The acidified samples were digested by microwave digestion system model Mars CEM under a pressure $160 \mathrm{Psi}$, and temperature $190^{\circ} \mathrm{C}$. The vessels model XP 1500 were used in the microwave digest, the remaining residues were treated with concentrated per-chloric acid andnitric acid followed by dryness and finally, dissolve the sample in hydrochloric acid.

\subsection{Analytical Procedure}

The Applied method is a development of that used by Juhani, (2001) [13] and Jukka in (2010) [14] which was designed for determination of Ra-226 in water samples. The method was modified to be applied on hard rocks (monazite and uranium ore). Samples were digested and spiked with a tracer (Ba-133) at the start of the procedure for chemical yield. Separation of radium was done by two ion-exchange columns first for remove the contaminants such as uranium isotopes and the second column for separation and purified of radium isotope fractions (Figure 2). The elution containing both Ra-226 and Ba-133 was evaporated and dissolution with $10 \mathrm{ml}(0.5 \mathrm{M} \mathrm{HCl})$, then divided into two parts one for 


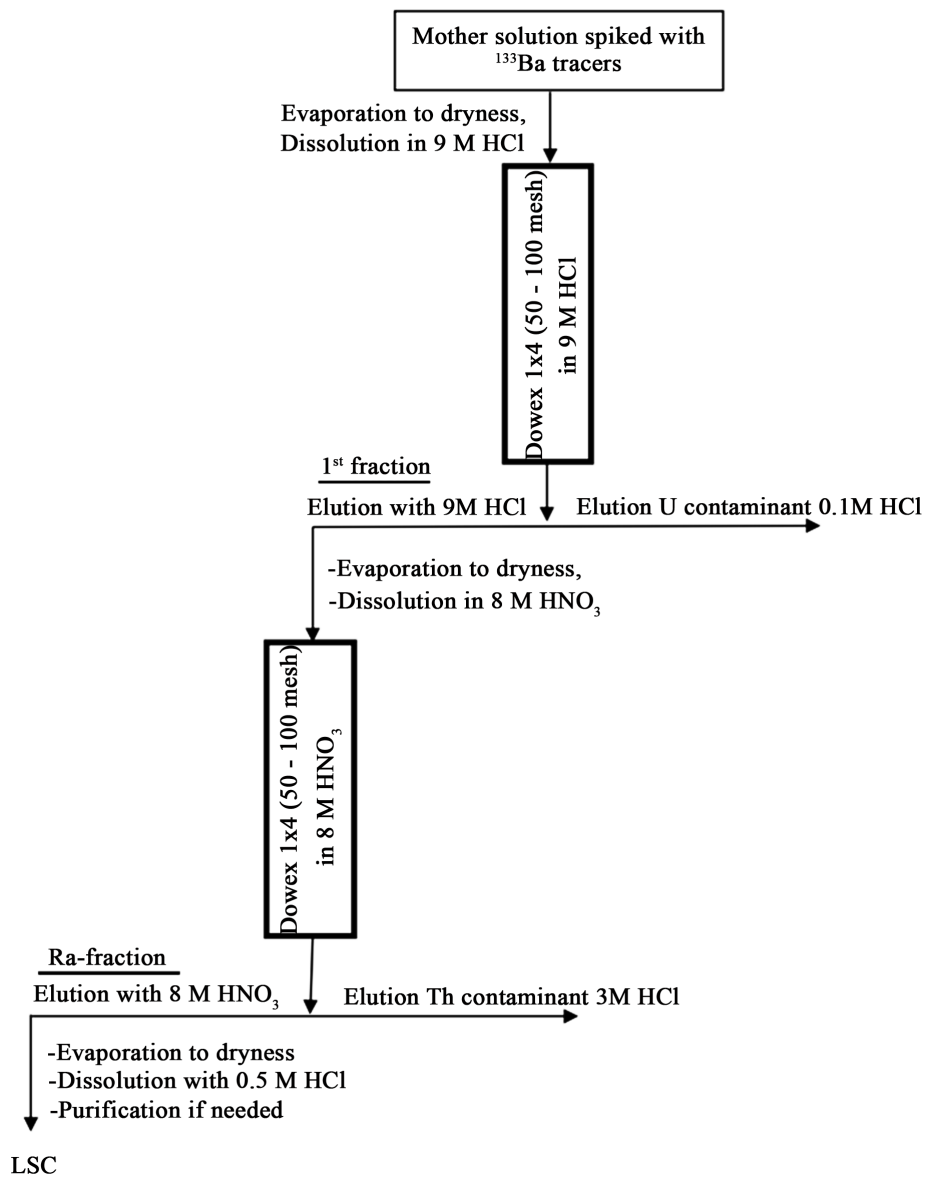

Figure 2. The schematic diagram of the radiochemical procedure.

counting by liquid scintillation counter and the other part for check the analysis by gamma spectrometry. Source preparation for counting by using barium nitrate as a carrier, $\mathrm{pH}$ adjusted to $\mathrm{pH} 2$ and $\mathrm{H}_{2} \mathrm{SO}_{4}$ was added $(3 \mathrm{ml})$ then the mixture was heated till boiling.

The solution cool at room temperature and filter through a Millipore filter, wash the precipitate three times with distilled water, removed the filter and allowed to dry for 30 minutes and counting by HPGe for determining the chemical recovery. The filter containing Ra-226 dissolved in $2 \mathrm{ml}$ basic EDTA and transferred to LSC vials for first counting (direct measured) then reserve 30 days for second measured.

\subsection{Standards Preparation}

Ba-133 standard was measured by high-resolution gamma spectroscopy at energy peak $356 \mathrm{keV}$ and by LSC to determine the chemical yield of the method. Ba-133 activities of the treated samples were then compared to the standard source prepared to calculate the chemical recovery. Figure 3(a) show the gamma spectrum of the Ba-133 standard source $8.3 \mathrm{~Bq}$ at counting time $600 \mathrm{~min}$. For using LSC, $2 \mathrm{ml}$ of the standard Ba-133 tracer evaporated to dryness and taken up in $2 \mathrm{ml}$ of EDTA, finally added $18 \mathrm{ml}$ of scintillation cocktail. The standards 


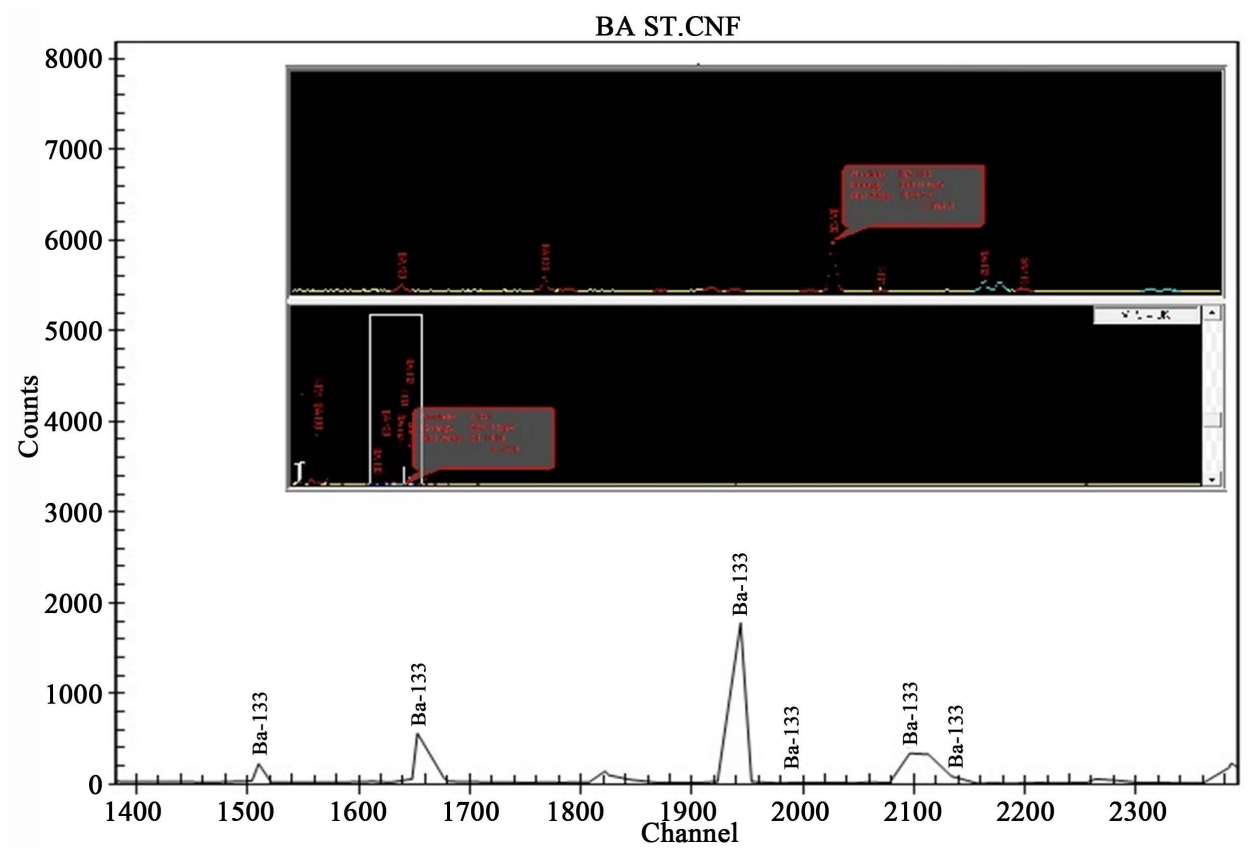

(a)

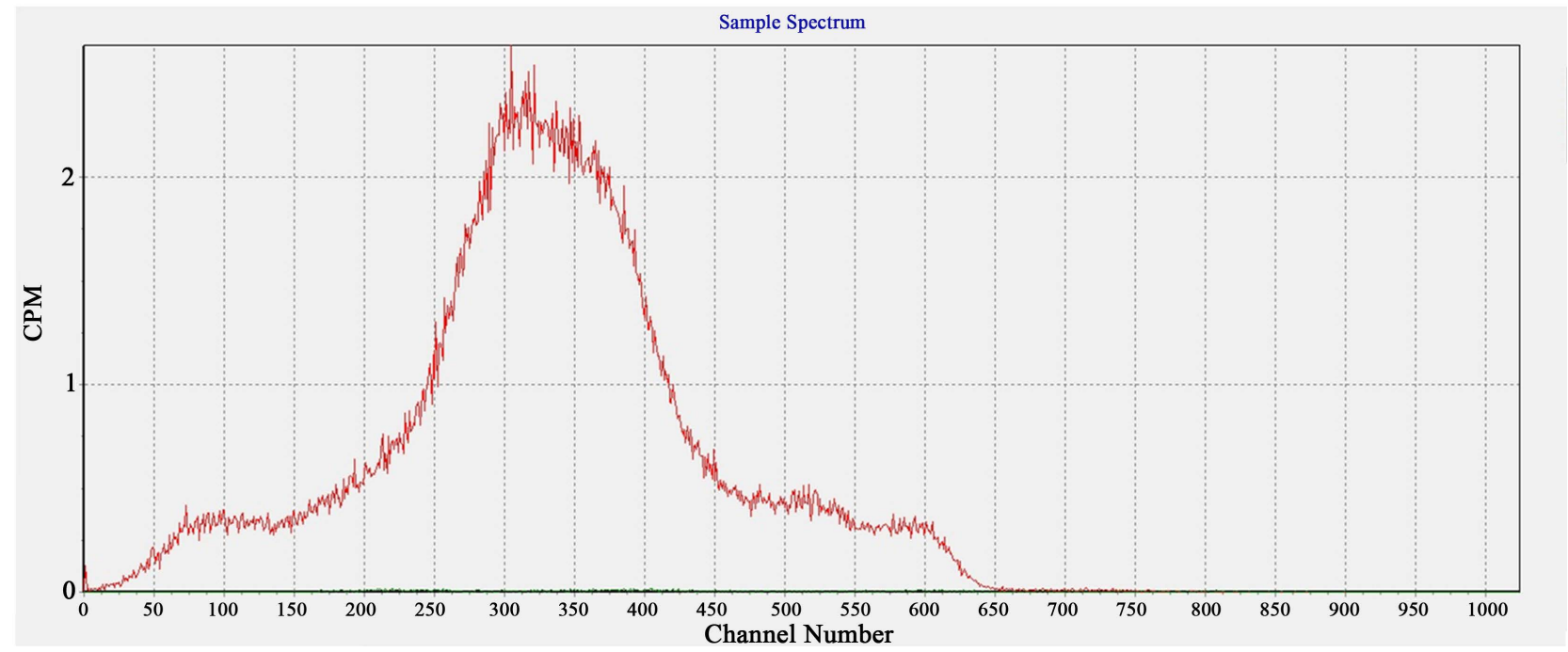

(b)

Figure 3. (a) Show high resolution gamma spectrum of the Ba-133 standard source $(8.3 \mathrm{~Bq} / \mathrm{vial})$ and counting time about 600 min; (b) Gamma spectrum of the Ba-133 standard source (8.3 Bq) in LSC.

were used to set the counting windows for future counting of Ba-133 for chemical recovery determination by LSC in this case it was found to be between channels 57 - 525. Figure 3(b) demonstrates the spectrum obtained by LSC for 8.3 $\mathrm{Bq} / \mathrm{vial} \mathrm{Ba}-133$.

\subsection{Instrumentation}

\section{- Gamma spectrometry}

Ba-133 activity were measured with low level gamma detector for calculating the chemical yield, Gamma ray spectrometer equipped with Hyper Pure Germa- 
nium (HPGe) detector was used. The gamma spectrometer consists of a detector of vertical configuration mounted on 30 liters liquid nitrogen dewar for temperature control. Supporting electronics i.e., the preamplifier, amplifier, ADC/MCA and high voltage power supply with positive polarity, are provided by Canberra DSA-1000, Desktop Spectrum analyzer. The used gamma ray spectrometer system was energy calibrated by ${ }^{152} \mathrm{Eu}$ source. The efficiency calibration is done by preparing several standard geometries from multi-nuclide standard solution number 7503 Eckert \& Ziegler $\left({ }^{210} \mathrm{~Pb},{ }^{241} \mathrm{Am},{ }^{109} \mathrm{Cd},{ }^{57} \mathrm{Co},{ }^{123 \mathrm{~m}} \mathrm{Tc},{ }^{51} \mathrm{Cr},{ }^{113} \mathrm{Sn},{ }^{85} \mathrm{Sr}\right.$, ${ }^{137} \mathrm{Cs},{ }^{88} \mathrm{Y}$ and ${ }^{60} \mathrm{Co}$ ). Geometry used in the present work is $15 \mathrm{ml}$ of sample in 20 $\mathrm{ml}$ Packard Liquid Scintillation polyethylene vials. Gamma spectrometry based on hyper pure germanium detectors was used with a relative efficiency $45 \%$ and full width at half maximum (FWHM) of $1.95 \mathrm{keV}$ for ${ }^{60} \mathrm{Co}$ gamma energy line at $1332 \mathrm{keV}$ and operated with Canberra Genie 2000 for gamma acquisition and analysis.

\section{- Liquid scintillation counter}

The Quantulus 1220 scintillation counter manufactured by Wallac, supported by Pulse-shape discrimination for $\alpha / \beta$ separation was used. The optimum settings for each standard counted individually at a range of Pulse Decay Discrimination, PDD setting and the percent misclassification of alpha into beta and vice versa are plotted against Pulse Decay Discrimination on the same graph for determination the optimum setting. Eight different Pulse Shape Analysis, PSA (50, $60,70,80,90,100,110$ and 120) levels were tested for Ra-226 determination using standard solutions of the same activity $(17.7 \mathrm{~Bq} /$ vial $)$ and composition of the measured standards like that intend to be measured at counting time $600 \mathrm{~min}$. The background is reduced by the presence of a Bismuth Germanium Oxide, BGO detector guard, which surrounds the counting chamber. The Gard detector utilized to measure the gamma radiation for Ba-133 yield tracer, for recovery calculation. Gamma pulses from Ba-133 show in the coincidence spectrum, the reliability of yield determination is ensured by the simultaneous detection of the yield tracer and radionuclide as explained by L. Saarinen et al., in 1992 [10].

\section{Results and Discussion}

The optimization of barium content is very important to find the best weight added from barium for the co-precipitation process. The amount of barium as $\mathrm{BaNO}_{3}$ was varied from 50, 100, 150, 200, 250, 300, $350 \mu \mathrm{g}$. Activities of the samples were then measured to study the relation between chemical recovery percent and the barium carrier ( $\mu \mathrm{g}$ ) added (Table 1). Barium sulphate co-precipitation technique is relatively cheap and has been widely used. The method based upon the similar chemical properties and behavior of isoelectric alkaline earth metals, barium and radium. Radium may be replacing barium in the crystal lattice due to their relatively nearly ionic radii $\mathrm{Ba}^{2+}=1.42 \AA$ and $\mathrm{Ra}^{2+}=1.48 \AA$ [12] [15]. Table 1 illustrate the Ba-133 recovery percent that determined by Gamma spectrometry for different masses of barium carrier added. The energy peak $356 \mathrm{keV}$ was used to calculate the chemical recovery for counting time $3 \mathrm{~h}$. 
Table 1. Ba-133 recovery percent and the amount of barium carrier in $\mu \mathrm{g}$ added at counting time $3 \mathrm{~h}$.

\begin{tabular}{ccc}
\hline $\begin{array}{c}\text { Mass of Barium } \\
(\mu \mathrm{g})\end{array}$ & $\begin{array}{c}\text { Gamma Measured at } 356 \mathrm{keV} \\
(\text { Counts })\end{array}$ & $\begin{array}{c}\text { Ba-133 Recovery } \\
(\%)\end{array}$ \\
\hline (0) Standard & $11673.4 \pm 105$ & 100.00 \\
50 & $6140.21 \pm 131.5$ & $52.6 \pm 3.01$ \\
100 & $7062.41 \pm 142.2$ & $60.5 \pm 4.3$ \\
150 & $8253.09 \pm 155.23$ & $70.7 \pm 5.6$ \\
200 & $9455.45 \pm 167.2$ & $81 \pm 6.4$ \\
250 & $11101.40 \pm 131.5$ & $95.1 \pm 3.8$ \\
300 & $8836.76 \pm 140.95$ & $75.7 \pm 3.9$ \\
350 & $11486.63 \pm 142.5$ & $98.4 \pm 4.09$ \\
\hline
\end{tabular}

The results in Table 1 illustrated that the recovery percent increase with increasing the mass of barium carrier. The solubility product constant $K_{s p}$ is a useful parameter for study the mechanism of the reaction by calculating the aqueous solubility of sparingly soluble compounds under various conditions. $K_{s p}$ $=\left[\mathrm{M}^{+}\right]^{\mathrm{m}}\left[\mathrm{A}^{-}\right]^{\mathrm{n}}$ is the equilibrium constant for the reaction. $K_{s p}$ is $1.08^{\star} 10^{-10}[16]$.

$$
\begin{gathered}
\mathrm{BaSO}_{4}(\mathrm{~s}) \rightarrow \mathrm{Ba}^{2+}(\mathrm{aq})+\mathrm{SO}_{4}^{2-}(\mathrm{aq}) \\
K_{s p}=1.1 \times 10^{-10}=\left[\mathrm{Ba}^{2+}\right]\left[\mathrm{SO}_{4}^{2-}\right]
\end{gathered}
$$

The solubility data shows that the amount of barium that might dissolve in $1 \mathrm{~L}$ of water is about $0.00137 \mathrm{~g}$. The solubility of $\mathrm{BaSO}_{4}$ is $1 \times 10^{-5}$ mole/liter and it is not particularly soluble in water. The elaborate data agree with the obtained by authors Sill et al., in 1974 [17] who provide a details and instruction for the co-precipitation of radium with barium sulphate and the subsequent micro-filtration. If the sample contains marginal amounts of barium less than $50 \mu \mathrm{g}$ [18] like potable water, blanks or standards, and the previous co-precipitation was carried out only with lead, barium carrier is added in the range of 75 - 175 $\mu \mathrm{g}$ and this agree with the obtained results as in Figure 4. The samples with a carrier greater than $50 \mu \mathrm{g}$ appeared to produce a significant precipitate and higher recovery percent $98 \%$ reached at $350 \mu \mathrm{g}$.

Ba-133 chemical recovery percent was estimated by comparison of the measured activities with the activities of the standard. Gamma spectrometry was carried out by measuring the counts under the peak at energy $356 \mathrm{keV}$ (counting time $3 \mathrm{~h}$ ). For LSC the activity between channels 75 - 525 in the gamma coincidence spectrum was used and compared with standard at counting time $600 \mathrm{~min}$ as demonstrated in Table 2 \& Figure 3(b).

Table 2 proves a large variation in the chemical recovery obtained from LSC and gamma spectrometry. The co-precipitation processes were made by added $250 \mu \mathrm{g}$ of Ba carrier and $5 \mathrm{ml} \mathrm{Ba}-133$ tracer with the activity $1.6 \mathrm{~Bq} / \mathrm{ml}$ in presence of Ra-226 spiked tracer. The calculated LSC chemical recovery reached in 


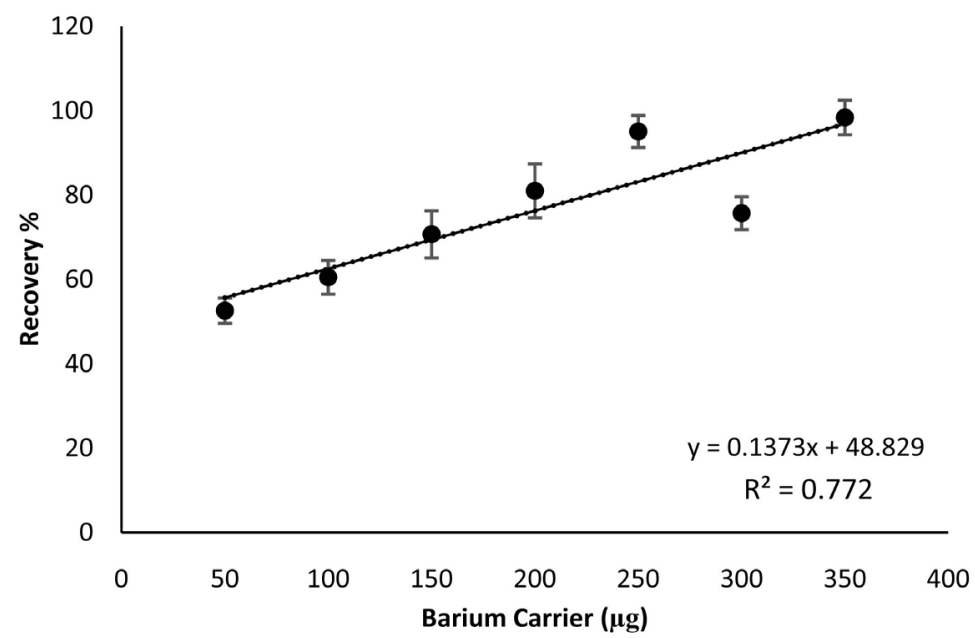

Figure 4. Variation of barium added in microgram versus the chemical recovery percent by gamma spectrometry.

Table 2. Chemical recoveries obtained from gamma spectrometry (HPGe) and LSC.

\begin{tabular}{cccccc}
\hline \multirow{2}{*}{$\begin{array}{c}\text { Sample } \\
\text { No. }\end{array}$} & $\begin{array}{c}\text { Ba-133 added } \\
(\text { Bq/Vial })\end{array}$ & $\begin{array}{c}\text { Gamma Spectrometry } \\
\text { Counts, }\end{array}$ & $\begin{array}{c}\text { Recovery } \\
(\%)\end{array}$ & $\begin{array}{c}\text { CPM, } \\
\text { In Time 600 min. }\end{array}$ & $\begin{array}{c}\text { Recovery } \\
(\%)\end{array}$ \\
\hline Standard & 8 & $11,101.4 \pm 31.5$ & & $33.5 \pm 2.30$ & \\
$\mathbf{1}$ & 8 & $10,458 \pm 92$ & 92 & $15.1 \pm 3.80$ & 45 \\
$\mathbf{2}$ & 8 & $10,582.5 \pm 100$ & 85 & $19.43 \pm 4.40$ & 58 \\
$\mathbf{3}$ & 8 & $8466 \pm 102$ & 77 & $25.13 \pm 5.01$ & 75 \\
$\mathbf{4}$ & 8 & $9835.5 \pm 90$ & 79 & $26.8 \pm 5.10$ & 80 \\
$\mathbf{5}$ & 8 & $7719 \pm 83$ & 62 & $23.45 \pm 4.80$ & 70 \\
$\mathbf{6}$ & 8 & $9586.5 \pm 70$ & 77 & $11.725 \pm 3.40$ & 35 \\
\hline
\end{tabular}

some samples very low percent such as $45 \%$ and $35 \%$ means that the results obtained of Ra-226 activity is not reliable So, gamma spectrometry is better for calculating the chemical yield according to the recovery percent noted in Table 2.

\subsection{Determination of Ra-226 Activity in Rock}

The obtained results compared with the known activity Ra-226, $1.6 \pm 0.05 \mathrm{~Bq} / \mathrm{g}$ spiked with $\mathrm{Ba}-133$ tracer for chemical recovery determination. The procedure was tested in different monazite samples in presence of certified reference material number DL-1a [19], Canadian certified reference materials. Chemical yield (Recovery \%) were measured twice at equilibrium and immediately after the purification of Ra-226 to prevent the ingrowths of the daughters that may be affecting on the results [10]. The specific activity Bq/g was calculated by Equation (1) and results presented in Table 4.

$$
\operatorname{Specific} \operatorname{Activity}(\mathrm{Bq} / \mathrm{g})=\frac{\operatorname{Corrected} \operatorname{activity}(\mathrm{Bq})}{\text { Mass of }(\text { rock }) \text { sample in }(\mathrm{g})}
$$


Pulse Decay Discrimination (PDD) setting and factors that affected on the activity were determined; to optimize alpha/beta separation performance where there is equal and minimum spill of alpha pulses into the beta Multi-Channel Analyzer, MCA and beta pulses into the alpha MCA [20].

\subsection{Factors Affecting on the Activity Determination by LSC}

\section{Quenching}

The misclassification is determined at the Pulse Decay Discrimination (PDD) setting. Plot the percentage misclassification versus transformer spectral index of the sample, tSIE for single PDD setting. The correction for misclassification as a function of quench is studied. Figure 5 illustrate the effect of Spectral Quench Parameter(SQP) on percent misclassification of alpha and beta events using $\mathrm{CCl}_{4}$ at different concentration $(0,50,100,150,200 \mu \mathrm{l})$ with the same activity 17.8 $\mathrm{Bq} / \mathrm{ml}$. Table 3 \& Figure 6 depicts the Alpha beta discrimination determined for Ra-226, Ba-133 standards and sample activity at different PSA values. The calculated results show that with increasing the concentration of $\mathrm{CCl}_{4}$ from 0 to $200 \mu \mathrm{l}$

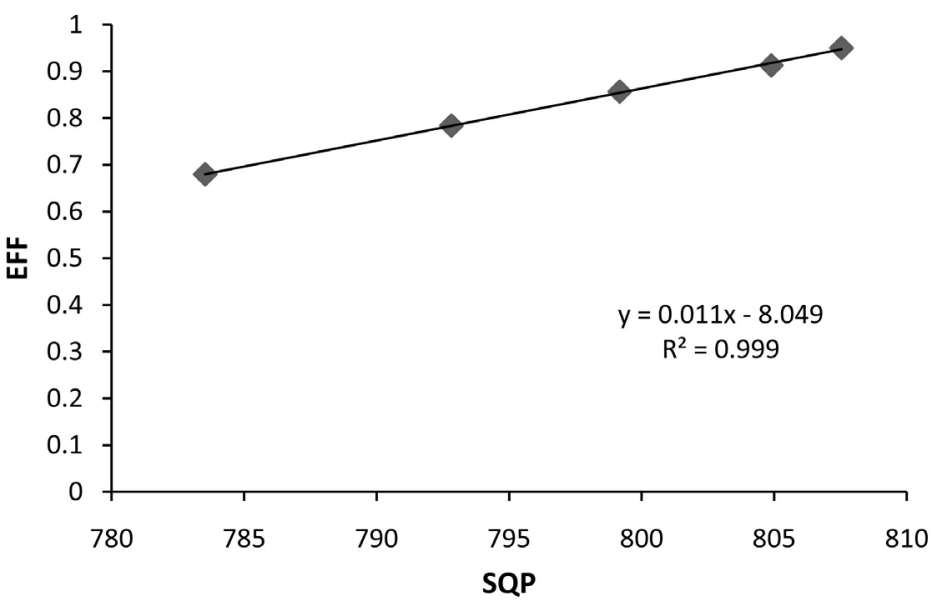

Figure 5. Correlation between Spectral Quench Parameter (SQP) and efficiency (EFF) at 100 Pulse Shape Analysis (PSA).

Table 3. Ra-226 activity (CPM) in the presence of quench solution $\mathrm{CCl}_{4}$ at different volume.

\begin{tabular}{|c|c|c|c|c|c|c|c|c|}
\hline \multirow{3}{*}{$\begin{array}{c}\mathrm{CCl}_{4} \\
\text { Added }(\mu \mathrm{l})\end{array}$} & \multicolumn{8}{|c|}{ Ra-226 (17.7 Bq/Vial) Activity (CPM) } \\
\hline & \multicolumn{8}{|c|}{ PSA } \\
\hline & 50 & 60 & 70 & 80 & 90 & 100 & 110 & 120 \\
\hline 0 & 40,414 & 80.92 & 75.95 & 72.88 & 68.90 & 66.53 & 62.76 & 57.78 \\
\hline 50 & 38,153 & 79.74 & 74.85 & 71.54 & 67.23 & 63.91 & 58.60 & 51.76 \\
\hline 100 & 35,255 & 79.82 & 74.21 & 69.43 & 65.77 & 59.98 & 52.57 & 42.93 \\
\hline 150 & 33,165 & 78.46 & 71.82 & 67.43 & 62.012 & 54.88 & 45.11 & 34.78 \\
\hline 200 & 27,975 & 76.90 & 69.50 & 63.30 & 56.06 & 46.76 & 35.69 & 24.88 \\
\hline
\end{tabular}

PSA = Pulse Shape Analysis. 


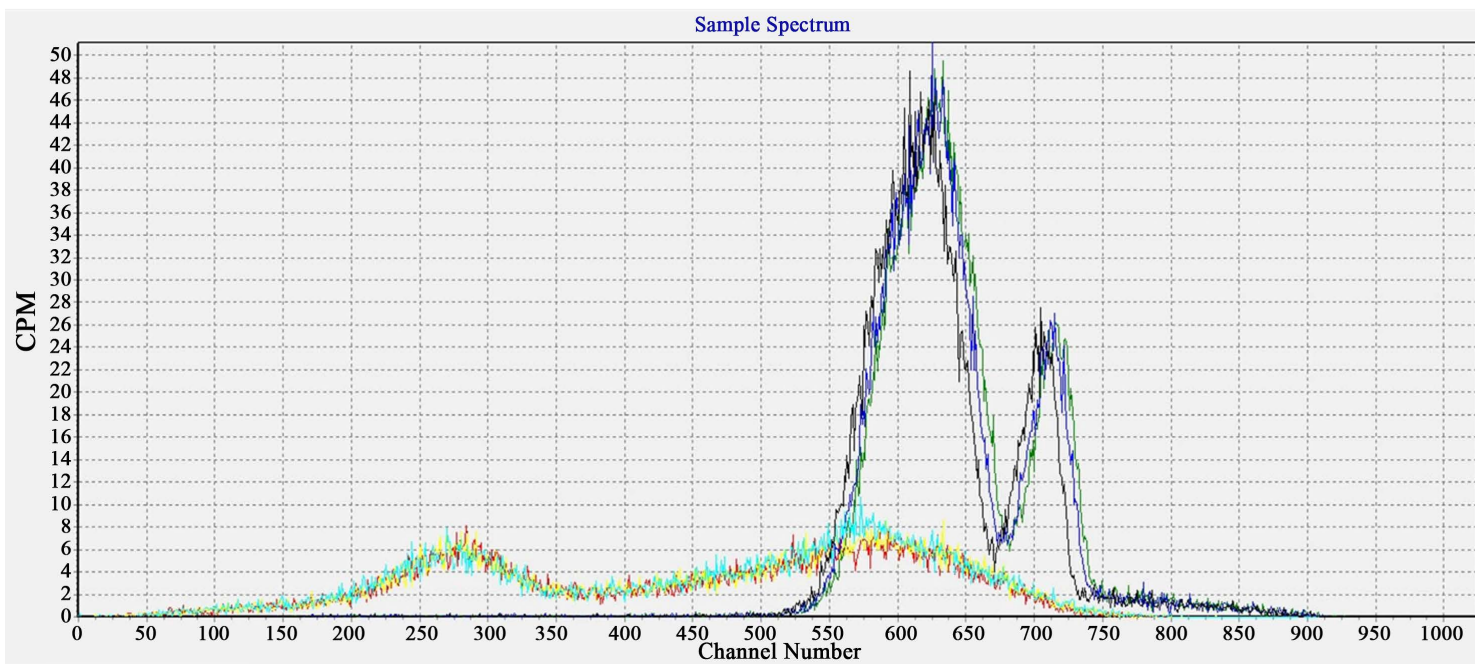

(a)

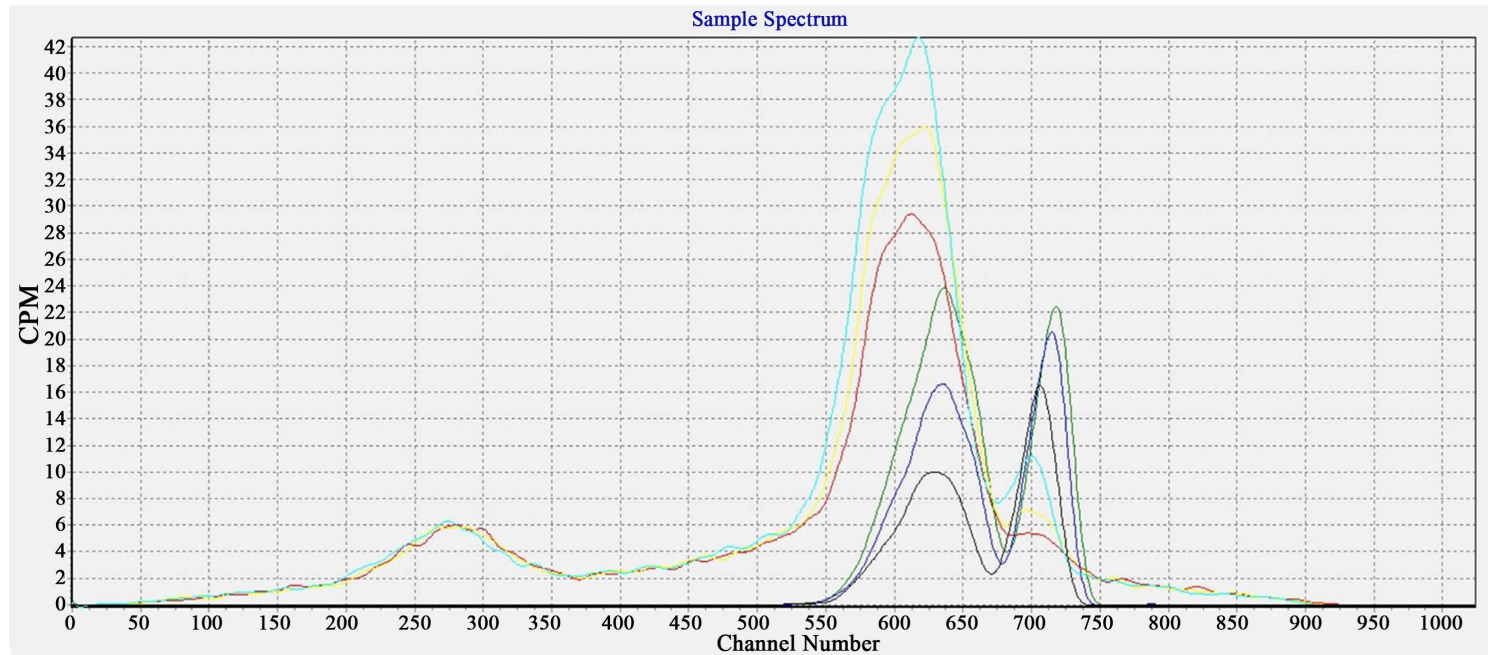

(b)
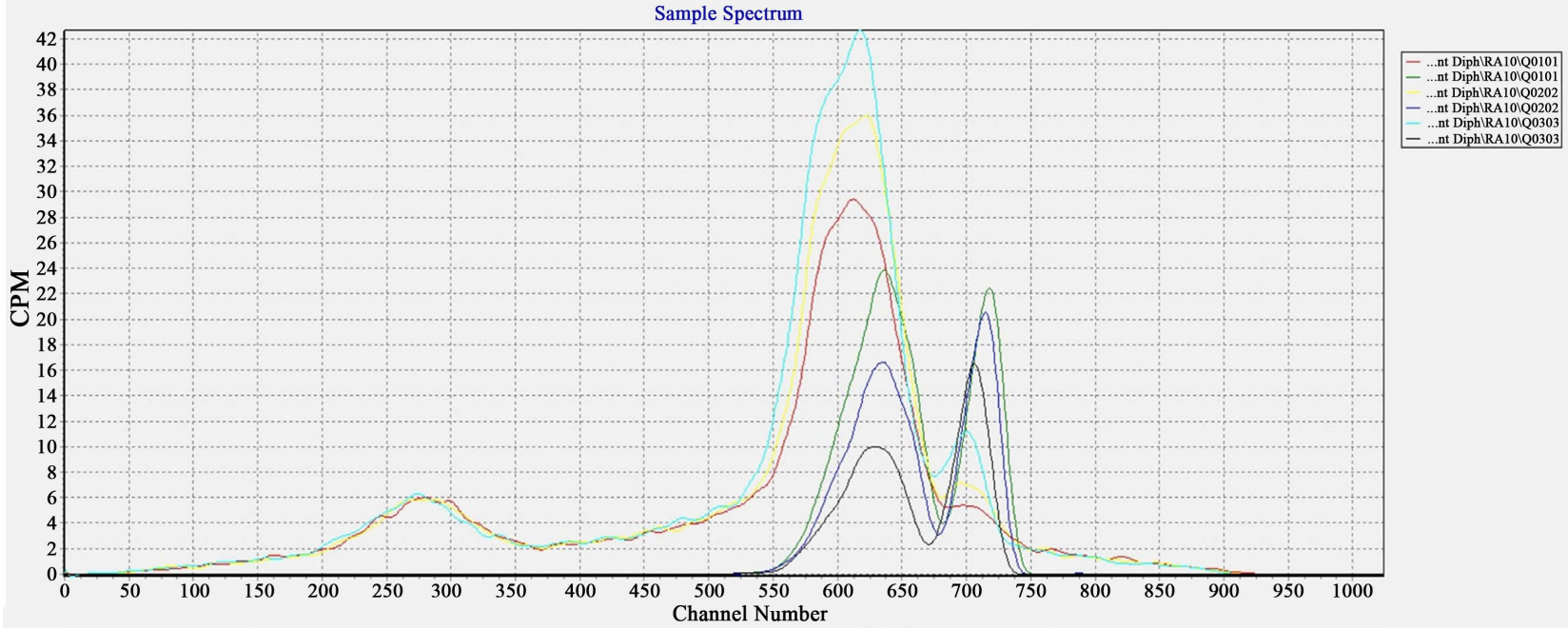

(c)

Figure 6. Quantulus alpha beta discrimination for Ra-226 \& Ba-133 at different PSA values, (a) Original curve; (b) Fitted curves and (c) Measured monazite sample. 
the activity determined for Ra-226 decrease due to the quench effect as illustrated in Table 3 and this results agree with that obtained by another investigators IAEA in 1990 [12], 2014 [15] and Charles et al., in 1994 [21].

\section{Materials of vial}

The background were measured by using polyethylene, PTFE vials (Zinsser). The measured counts were 2 CPM and 6 CPM for alpha and beta respectively it was lower than the counts in case of using glass materials which measured 3 CPM and 16 CPM for alpha and beta. The polyethylene vials were chosen as the best option for display lower background as recommended from other authors Yu-Yufu et al., (1990) [22] and IAEA, (2010) [1].

\section{Sample volume}

The volume of samples was kept constant during the measurement $5 \mathrm{ml}$ and $15 \mathrm{ml}$ of cocktail. The obtained results from using different volumes of liquid scintillation cocktails were studied and concluded that the best volumes are the minimum volume due to the lower quench effect [23].

Spectral Quench Parameter (SQP) is used at 100 PSA for determination the efficiency used in the calculation of unknown samples as depicted in Figure 5.

Figure 6 show the spectrum of samples contains some impurities which may be the reason for peak shift for Po-214 around channel 720 in the trace spectrum, to be 707. It has been observed from previous studies that the Po-214 peak can become indistinguishable from the large peak that may be obtained, hence making the determination of Ra-226 very difficult and this agree with another investigators Juutunen et al., in 2001 [11]. Table 4 shows that Ra-226 activities determination for the certified reference materials (ore number DL-1a) and monazite rock by LSC at counting time $500 \mathrm{~min}$. The obtained results have a little sign of quenching and self-absorption in the spectra than the purified samples.

The average chemical recovery determined for certified reference material number DL-1a was $89.40 \%$ for the average activity $1.27 \pm 0.15 \mathrm{~Bq} / \mathrm{g}$ and the results were relatively near the values in the certificate $1.4 \pm 0.04 \mathrm{~Bq} / \mathrm{g}$ [24]. The

Table 4. Ra-226 activities of certified reference materials (DL-1a) and monazite by LSC at counting time $500 \mathrm{~min}$.

\begin{tabular}{ccccc}
\hline Sample Code & Mass (mg) & Ba-133 Recovery \% & Count Rate (CPM) & Activity (Bq/g) \\
\hline DL-1a & 43 & $90.10 \pm 9.10$ & $6.38 \pm 0.32$ & $1.27 \pm 0.15$ \\
DL-1a & 44 & $88.70 \pm 10.3$ & $6.16 \pm 0.31$ & $1.26 \pm 0.14$ \\
Average & 43.5 & $89.40 \pm 9.65$ & & $1.27 \pm 0.15$ \\
Monazite 1 & 51 & $78.88 \pm 5.5$ & $71.16 \pm 5.11$ & $13.96 \pm 1.38$ \\
Monazite 2 & 53 & $80.30 \pm 4.6$ & $72.50 \pm 3.1$ & $14.22 \pm 1.20$ \\
Monazite 3 & 50 & $79.40 \pm 4.8$ & $71.60 \pm 4.7$ & $14.05 \pm 1.25$ \\
Monazite 4 & 52 & $74.4 \pm 5.1$ & $67.12 \pm 5.9$ & $13.17 \pm 4.4$ \\
Average & 51.5 & $78.24 \pm 5$ & & $13.85 \pm 2.05$ \\
Ra-St \& Ba-St & & $\sim 100$ & $90.21 \pm 1.07$ & $18.1 \pm 1.11$ \\
\hline
\end{tabular}


variation in the chemical recovery in Monazite samples were varied from $74.4 \%$ to $80.3 \%$ as shown in Table 4 and this may be due to the long steps for the radiochemical separation of the contaminants (Uranium and Thorium isotopes). The relatively small quantity of radium present in geological samples play a role in chosen the preferable mechanism that may be co-precipitation and adsorption.

Major natural sulphate in the samples may cause premature precipitation, thus preventing interchange of radium and barium in $\mathrm{BaSO}_{4}$ crystal lattice. Adsorption reactions of radium can be obstruction by substances present in natural samples and this agree with Golden et al., 1961 [25].

The good chemical recovery for the DL-1a samples using Ba-133 standard solutions reach about $90.10 \%$ (Table 4) and this attribute to the homogeneous in the ore and the counting in $4 \Pi$ geometry by LSC. The relatively low chemical recovery \% in monazite reach $74.4 \%$ in monazite 4 and this attributed to the color of the samples that cause the quench effect as explained by, Wallac LKB in 1991 [26]. In addition, Ba-133 used as a tracer for Ra-226 determination under very specific conditions that may cause a little effect comparing with $\mathrm{Ra}-225$ trace. Pulse shape discrimination applied to reduce the backgrounds by rejecting beta and gamma pulses with $99.95 \%$ efficiency. Apply of Photon Electron Rejecting Alpha Liquid Scintillation (PERALS) indicate that the resolution of 200-250 keV (FWHM) and background about 0.01 CPM can be achieved as identified by Purkel \& Eisenhauer in 2003 [3] and Volpe et al., in 1991 [27].

$$
\operatorname{LLD}(\mathrm{Bq} / \mathrm{g})=\frac{4.66 \sqrt{\mathrm{BG}}}{t \cdot \varepsilon \cdot y \cdot m}
$$

where, BG.: The blank counts under the peak of interest, $t$. Counting time in second, $\varepsilon$. Efficiency of detector LSC ( $100 \%), y$. The average yield obtained, $m$ : The mass of sample in $(\mathrm{g})$ and 4.66: Is a statistical factor for one tailed distribution required.

The calculated LLD according to Equation (2) [28] is $9.5 \mathrm{mBq} / \mathrm{g}$ for Ra-226 in monazite samples and this may agree with another authors who reported the values about LLDs for radium isotopes in different matrixes IAEA, 2010 [29] and Chalupnik et al., 1993 [30].

\section{Conclusion}

The analytical procedure for the determination of Ra-226 in hard rocks, such as monazite and certified reference material number (DL-1a) has been modified. Based on the results, it can be concluded that the utilization of liquid scintillation counter in the determination of Ra-226 in samples was found to be quick and versatile with a high accuracy, low limit of detection $9.5 \mathrm{mBq} / \mathrm{g}$ with an average chemical recovery $78.2 \%$ and $89.4 \%$ for monazite samples and certified reference material respectively. The proposed method can be readily used due to the availability of the instrumentation, the low cost of analysis and the required sensitivity. Finally, the obtained results may shed some light on the liquid scin- 
tillation counter applications and provide more options for radiochemists and decision makers to select the suitable technique which meets the analyses needs.

\section{Acknowledgements}

With gratitude the author acknowledges the contribution of the Helsinki university radiochemical laboratory, students, their teachers, as well as the scientific guidance from Dr. Kerttuli Helariutta, Dr. Juhani Suksi and Prof. JukkaLehto to the present study. The work was funded by ParOwn (Project No. 15326).

\section{Conflicts of Interest}

The author declares no conflicts of interest regarding the publication of this paper.

\section{References}

[1] International Atomic Energy Agency (2010) Analytical Methodology for the Determination of Radium Isotopes in Environmental Samples. Analytical Quality in Nuclear Applications, No. IAEA/AQ/19.

[2] Martin, P. and Hancock, G.J. (2004) Routine Analysis of Naturally Occurring Radionuclides in Environmental Samples by Alpha-Particle Spectrometry. Supervising Scientist Report 180, Supervising Scientist Division, Darwin NT.

http://www.environment.gov.au/ssd/publications/ssr/180.html

[3] Purkl, S. and Eisenhauer, A. (2003) A Rapid Method for $\alpha$-Spectrometric Analysis of Radium Isotopes in Natural Waters Using Ion-Selective Membrane Technology. Applied Radiation and Isotopes, 59, 245-254. https://doi.org/10.1016/S0969-8043(03)00172-6

[4] Benedik, L., Spasova, Y., Vasile, M. and Atjen, U.W. (2008) Nuclear and Radiochemistry (NRC-7). Proceedings of the 7 th International Conference on Nuclear and Radiochemistry, Budapest, Hungary, 24-29 August 2008, 320.

[5] Jobbágy, V., Kávási, N., Somlai, J., Dombovári, P., Kardos, R. and Kovács, T. (2010) Radioanalytical Investigations of Uranium Concentrations in Natural Spring, Mineral, Spa and Drinking Waters in Hungary. Journal of Radioanalytical and Nuclear Chemistry, 286, 417-422. https://doi.org/10.1007/s10967-010-0711-5

[6] Tinker, R.A., Smith, J.D. and Cooper, M.B. (1995) An Assessment of the Selection criteria for an Analytical Method for Radium-226 in Environmental Samples. Journal of Radioanalytical and Nuclear Chemistry, 193, 329-336. https://doi.org/10.1007/BF02039890

[7] Lim, T.P. and Dave, N.K. (1981) A Rapid Method of Radium-226 Analysis in Water Samples Using Alpha Spectroscopic Technique. CIM Bulletin, 74, 97-105.

[8] Gomez Escobar, V., Vera Tome, F. and Lozano, J.C. (1999) Extractive Procedure for ${ }^{226} \mathrm{Ra}$ Determination in Aqueous Samples by Liquid Scintillation Counting. Radioactivity and Radiochemistry, 10, 17-21.

[9] Manjon, G., Vioque, I., Moreno, H., Garcia-Tenorio, R. and Garc, M. (1997) Determination of Ra-226 and Ra-224 in Drinking Water by Liquid Scintillation Counting. Applied Radiation and Isotopes, 48, 535-540. https://doi.org/10.1016/S0969-8043(96)00297-7

[10] Saarinen, L. and Suksi, J. (1992) Determination of Uranium Series Radionuclides Pa-231 and Ra-226 by Liquid Scintillation Counting, Nuclear Waste Commission of 
Finnish Power Companies. Technical Report, Helsinki, Finland, October 1992 (YJT-95-01).

[11] Juutunen, P., Ruutu, A. and Suksi, J. (2001) Determination of Ra-226 from Rock Samples Using LSC. Internal Report, University of Helsinki, Finland.

[12] International Atomic Energy Agency (1990) The Environmental Behavior of Radium. Technical Report Series, Vienna, TECDOC No. 310.

[13] Suksi, J. (2001) Natural Uranium as a Tracer in Radionuclide Geosphere Transport Studies. Report Series in Radiochemistry, University of Helsinki, 16/2001.

[14] Jukka, L. and Hou, X. (2010) Chemistry and Analysis of Radionuclides, Laboratory Techniques and Methodology. Wiley, New York, 69-71.

[15] International Atomic Energy Agency (2014) A Procedure for the Rapid Determination of Ra-226 and Ra-228 in Drinking Water by Liquid Scintillation Counting. Vienna, Series, AQ/39.

[16] Lide, D.R. (2003) HandBook of Chemistry and Physics. 84th Edition, CRC Press, Boca Raton, FL.

[17] Sill, C.W., Puphal, K.W. and Hindman, F.D. (1974) Simultaneous Determination of Alpha Emitting Nuclides of Radium through Californium in Soil. Analytical Chemistry, 46, 1725-1737. https://doi.org/10.1021/ac60348a021

[18] Bojanowski, R., Radecki, Z. and Burns, K. (2005) Determination of Radium and Uranium Isotopes in Natural Waters by Sorption on Hydrous Manganese Dioxide Followed by Alpha-Spectrometry. Journal of Radioanalytical and Nuclear Chemistry, 264, 437-443. https://doi.org/10.1007/s10967-005-0734-5

[19] Smith, C.W. and Steger, H.F. (1983) Radium-226 in Certified Uranium Reference Ores Dl-1a BL-4a, DH-1a and BL-5. Canada Central for Mineral Energy Technology, Ottawa, Canada.

[20] Mien, N.Q. and Loat, B.V. (2008) Determination of the Annual Beta Dose by Measuring Beta Activity Using the Liquid Scintillation Technique. VNU Journal of Science, Mathematics-Physics, 24, 36-41.

[21] Passo, C.J. and Cook, G.T. (1994) Handbook of Environmental of Liquid Scintillation Counter, LSC Spectrometry, Packard Instrument Company, Meriden, CT.

[22] Yufu, Y., Salbu, B., Bjoernstad, H.E. and Lien, H. (1990) Improvement for $\alpha$-Energy Resolution in Determination of Low-Level Plutonium by Liquid Scintillation Counting. Journal of Radioanalytical and Nuclear Chemistry, 45, 345-353. https://doi.org/10.1007/BF02165075

[23] Kleinschmidt, R.I. (2004) Gross Alpha and Beta Activity Analysis in Water-A Routine Laboratory Method Using Liquid Scintillation Analysis. Applied Radiation and Isotopes, 61, 333-338. https://doi.org/10.1016/j.apradiso.2004.03.004

[24] Canada Center for Minerals and Energy Technology (2017) Reference Uranium and Thorium Ore. Energy of Mines and Resources Canada, Number DL-1a. http://www.nrcan.gc.ca/mining-materials/certified-reference-materials/certificate-p rice-list/8049

[25] Goldin, A.S. (1961) Determination of Dissolved Radium. Analytical Chemistry, 33, 406-409. https://doi.org/10.1021/ac60171a030

[26] QuantulusWallac, L.K.B. (1991) Pulse Shape Analysis in Liquid Scintillation Counting. Wallac Application Product News, November 1991.

[27] Volpe, A.M., Olivares, J.A. and Murrell, M.T. (1991) Determination of Radium Isotope Ratios and Abundances in Geologic Samples by Thermal Ionization Mass Spectrometry. Analytical Chemistry, 63, 913-916. 
https://doi.org/10.1021/ac00009a015

[28] Currie, L.A. (1968) Limits for Detection and Quantitative Determination. Analytical Chemistry, 40, 586-593. https://doi.org/10.1021/ac60259a007

[29] Cook, M.L. and Kleinschmidt, R. (2009) Simultaneous Measurement of Ra-226 and Ra-228 in Water by Liquid Scintillation Method. Queensland Health, Australia.

[30] Chalupnik, S. and Lebecka, J.M. (1993) Determination of Ra-226, Ra-228 and Ra-224 in Water and Aqueous Solutions by Liquid Scintillation Counting. International Conference on Advances in LSC. Radiocarbon, 397-403. 